\title{
Mimesis et politique dans Representative Men de R. W. Emerson
}

\author{
Thomas CONSTANTINESCO \\ Université Paris Diderot \\ LARCA (EA 4214)
}

Notice biographique: Thomas Constantinesco est maître de conférences à l'université Paris Diderot. Il est l'auteur de Ralph Waldo Emerson. L'Amérique à l'essai (2012), ainsi que de plusieurs articles sur Emerson et la littérature américaine du XIX ${ }^{\mathrm{e}}$ siècle. Il a également dirigé avec Antoine Traisnel Littérature et Politique en Nouvelle-Angleterre (2011).

Mots-clés: Emerson ; Representative Men ; mimesis ; représentation ; philosophie ; politique.

Keywords: Emerson; Representative Men; mimesis; representation; philosophy; politics.

Abstract: Emerson's Representative Men (1850) weaves together aesthetics and politics as it addresses the civic role of the poet. This article shows how Emerson repeats Plato's inaugural determination of mimesis as imitation the better to expose the failure of its logic and offer an alternative form of mimesis whose effects shape the community to come.

$$
* * *
$$

Représenter l'Amérique dans le champ littéraire et sur la scène politique, tel est le défi que lance Emerson dans Representative Men, où la représentativité des grands hommes que désigne le titre semble dériver de leur puissance esthétique : «Men have a pictorial or 
representative quality » (618), annonce-t-il d'entrée de jeu. Il s'agit donc de donner à la nation forme et figure et en même temps de parler en son nom, d'inventer la communauté tout en se faisant l'écho de la volonté générale. Or, pour être des faiseurs d'images, pour être passés maîtres dans l'art de la mimesis, les artistes seraient les plus grands héros et les mieux à même de servir de représentants du peuple au Congrès. La capacité de figuration qu'ils possèdent les rendrait aptes à participer au gouvernement de la cité. En un mot, leur savoir mimétique légitimerait leur action politique ${ }^{1}$.

À l'instar de The Scarlet Letter et Moby Dick dont il est contemporain, Representative Men interroge à nouveaux frais la relation entre mimesis et politique depuis la scène d'écriture de la «Renaissance américaine ». Issu d'une série de conférences commencée en 1845, ce recueil est publié en janvier 1850 et prend acte d'une crise de la représentation, aux sens politique et esthétique du terme. Le constat n'est pas neuf : l'élection d'Andrew Jackson en 1828 et l'avènement de la « démocratie populaire » ont marqué pour Emerson les premiers signes d'une regrettable corruption de l'esprit public, tandis que Nature (1836) et «The American Scholar» (1837) posaient déjà la redoutable question d'une production artistique autochtone et autonome qui ne se réduirait pas à la simple imitation des œuvres européennes. C'est dans ce contexte proprement critique qu'Emerson réfléchit aux modalités de la représentation en Amérique et qu'il procède, c'est du moins l'une des hypothèses de cette étude, à une relecture du concept de mimesis tel que la Vieille Europe l'aura transmis au Nouveau Monde.

Cette entreprise de relecture de la mimesis prend toutefois d'emblée des allures paradoxales, car Representative Men se présente comme une réponse à l'ouvrage de Carlyle, On Heroes, Hero Worship and the Heroic in History (1841). Il en reprend non seulement la forme, celle de la série d'esquisses biographiques, mais aussi plusieurs figures, comme Shakespeare ou Napoléon, et même certains concepts, tel l'« usage» du héros. Pris dans le 
piège mimétique qu'il n'a cessé de dénoncer, Emerson paraît ne pouvoir s'émanciper des lettres européennes qu'au prix de leur imitation et la naissance tant espérée d'une littérature nationale ressemble au bout du compte à la reprise d'une œuvre antérieure. Or cette compulsion de répétition est d'autant plus remarquable que, contre toute attente, aucun des «représentants » que propose cette galerie de portraits n'est américain et aux côtés de Swedenborg, Montaigne, Napoléon et Goethe, on trouve Platon et Shakespeare qui auront marqué l'histoire de la mimesis sur ses versants philosophique et esthétique. D'un texte à l'autre cependant, un écart décisif se creuse, car le «héros »s'efface devant l'«homme représentatif » et la célébration d'une aristocratie de demi-dieux est remplacée par l'élection de figures démocratiques. Ce faisant, Representative Men transfigure l'absence de représentants natifs en ressort d'une représentation politique et littéraire qui échapperait à la loi de la reproduction : puisqu'il ne saurait être question d'imiter des modèles européens, les offrir malgré tout à titre de représentants devient une manière de suggérer que la représentation procède d'une mimesis qui n'imite rien, sinon ce qui advient dans le mouvement même de la représentation ${ }^{2}$.

Representative Men est ainsi traversé par une tension entre deux modes de représentation de la représentation elle-même : la première, que l'on pourrait qualifier de platonicienne, reconduit à l'imitation, tandis que la seconde s'emploie à l'en dégager. Cette tension, qui empêche la mimesis émersonienne de coïncider avec elle-même, se donne à lire jusque dans le couplage de termes par lequel le texte désigne l'homme représentatif dont il promet l'avènement: «[a] poet-priest» (726). Le représentant se dédouble; il tient simultanément du démiurge façonnant le monde et du prophète colportant une parole qui lui préexiste. Interprète d'un savoir dont il n'est pas l'origine, ce prédicant séculier est appelé à révéler la destinée providentielle de la nation - destin tout tracé et joué d'avance, qu'il n'y aurait plus dès lors qu'à rejouer. Mais son ministère n'est pas sans ambiguïté, dans la mesure 
où le génie consiste pour le poète à s'inventer lui-même, libre de tout modèle, à réapparaître sous des traits toujours différents dans un jeu de masques sans fin. Si le grand homme fait fonction de «rassembleur» (a reconciler $[661,726])$, son identité demeure vicariante, à la fois exemplaire et exceptionnelle, et ce qui se rassemble en lui reste de l'ordre d'une différence inassimilable. Après avoir montré comment Representative Men reproduit dans un premier temps l'interprétation platonicienne de la mimesis pour mieux en diagnostiquer la faillite, il s'agira d'envisager l'autre mimesis qui sous-tend le recueil et qui, refusant d'ordonner la représentation à « la philosophie de l'identité » (the Identity-philosophy [668]), produit des figures qui ne sont représentatives que pour autant qu'elles diffèrent les unes des autres autant que d'elles-mêmes. Il faudra alors méditer sur le sens de cette différence, c'està-dire tenter de mesurer les effets d'une telle mimesis «native» sur la scène politique américaine.

\section{La philosophie de l'identité}

Si Representative Men répète la lecture classique de la mimesis dans le but de procéder à une critique de sa détermination platonicienne qui l'assimile à l'imitation, force est pourtant de constater que tout commence par un renversement apparent du platonisme. La philosophie, on le sait, s'inaugure avec Platon sur le mode d'un discours anti-mimétique et se fonde, au livre X de la République, sur l'expulsion rituelle du poète hors des limites de la cité. Or cette exclusion de l'art délimite simultanément le politique comme cet espace où seul le philosophe-roi a vocation à exercer le pouvoir (Lacoue-Labarthe, «Typographie » 227). En faisant du poète le représentant par excellence, Emerson place au contraire la mimesis au cœur même de la cité et inscrit la poursuite du bonheur de l'artiste parmi les priorités du 
gouvernement: «Society has really no graver interest than the well-being of the literary class » (750). Modèle de vertu civique, l'homme de lettres a rang d'homme de loi, et même, comme chez Shelley, de législateur (the lawgiver [750]). Pour parler comme Rancière, il est chargé d'opérer un nouveau «partage du sensible ${ }^{3}$ », lui qui par son don de visionnaire sait proposer du visible un nouvel agencement, ordonné selon les principes qu'il dégage de sa lecture des signes de la nature :

nature has more splendid endowments for those whom she elects to a superior office; for the class of scholars or writers, who see connection where the multitude see fragments, and who are impelled to exhibit the facts in order, and so to supply the axis on which the frame of things turns. (747)

Là où Platon scelle le contrat politique dans et par le sacrifice «pharmaceutique » du poète, Emerson décerne à l'écrivain le titre de citoyen d'honneur et le déclare garant de l'ordre public.

Ce renversement est toutefois moins une sortie du régime platonicien, et donc une sortie de l'imitation, que sa confirmation, dans la mesure où continuent de se nouer dans les deux cas la question de l'art et celle de la politique. Chez Platon, l'opprobre dont est victime le poète est le signe négatif de sa distinction et le même dispositif s'observe chez Emerson moyennant une simple inversion des signes électifs. Le retour du poète au sein de la communauté n'aura finalement servi qu'à reconduire la mimesis au fondement du politique. De fait, passé le chapitre introductif consacré aux « usages des grands hommes », le recueil se poursuit sous le haut patronage de Platon et de sa métaphysique de l'idée, avant d'évoquer Swedenborg et sa théorie des correspondances, puis de célébrer, un peu plus tard, le génie de Shakespeare et de terminer en vantant les mérites du Wilhelm Meister de Goethe, qui est 
aussi, entre autres choses, un roman sur Shakespeare et le théâtre. Au fil des différents chapitres, Emerson décèle la parenté secrète qui unit les grands noms de l'histoire de la mimesis et met au jour la lignée (the line of relation [661]) qu'a inaugurée Platon : « Hamlet is a pure Platonist [...]. Swedenborg, throughout his prose poem of "Conjugal Love," is a Platonist. » (659) Representative Men prend ainsi la forme d'un vaste portrait de famille, ou plutôt d'un immense arbre généalogique auquel se rattache naturellement la branche américaine, dernier rejeton d'une illustre dynastie de platoniciens. Moins linéaire que cyclique, l'histoire se répète de génération en génération et d'une rive à l'autre de l'Atlantique. C'est pourquoi Emerson peut dire de Platon qu'il est aussi le type même du génie américain, tout comme il incarne l'esprit national grec, anglais, allemand ou italien : «Plato seems to a reader in New England an American genius. His broad humanity transcends all sectional lines. »(634)

Puisque tous ont en partage ce glorieux aïeul, Platon prend les allures paradoxales d'une figure à la fois universelle et nationale. À ce titre, il peut constituer la pierre angulaire sur laquelle s'édifie la communauté et semble du même coup capable de garantir l'unité de la nation à un moment où, précisément, les « conflits de section » divisent le pays. Or, si Platon fait office de figure de l'unification, c'est d'abord parce que sa philosophie repose sur une métaphysique de l'idée, c'est-à-dire de l'identité et de l'unité. Dans la République, l'ordre politique est compris en termes ontologiques et la souveraineté y relève littéralement d'une hénologie, d'une science de l'un. Platon institue en effet, par la voix de Socrate, le philosophe-gouvernant comme cette figure idéale du pouvoir qui façonne la cité à son image en commandant la multitude des citoyens entendue au sens d'une multiplicité d'exemplaires semblables les uns aux autres. Évoquant le projet de Platon, Emerson suggère cependant que l'activité philosophique consiste en premier lieu à distinguer, différencier et définir chaque chose selon son essence : «This defining is philosophy. » (637) Mais si l'on rapproche les 
chapitres consacrés à Platon et Swedenborg, comme nous y invite l'architecture binaire du volume héritée des Vies Parallèles de Plutarque (Schulz), il apparaît que cette opération critique revient surtout à repérer des ressemblances essentielles (essential resemblances [677]) pour organiser le monde selon la logique du même (The Same, the Same [638]). De ce point de vue, Swedenborg apparaît comme l'héritier le plus fidèle de Platon, car la perception de l'identité est chez lui une qualité innée (that native perception of identity [668]) et toute son œuvre est un patient relevé des innombrables analogies qui unissent l'esprit et la matière. Le premier parvient à reconnaître dans la seconde le double de lui-même, dans la mesure où la nature est foncièrement symbolique. Ou plutôt, la nature est d'essence mimétique tant il est vrai qu'elle ne cesse de se répéter dans l'ensemble de ses productions, qui ne sont finalement que des reproductions : «Nature iterates her means perpetually on successive planes. In the old aphorism, nature is always self-similar»(668); «These grand rhymes or returns in nature [...] delighted the prophetic eye of Swedenborg ». (670) Même l'entreprise de traduction de ses œuvres en anglais par James John Garth Wilkinson ${ }^{4}$ est interprétée, selon la loi de la reproduction universelle, comme une manière pour Swedenborg de se dupliquer luimême (670-671). En vertu de son pouvoir quasi prophétique, qui lui permet de discerner les rapports d'homologie qui font l'unité du monde, il se fait l'interprète d'une nature qui parle en langage analogique. Avec lui, la nature s'exprime en images (in endless picture-language [674]), mais ses figures ne disent rien d'autre que sa propre identité : «Creative force, like a musical composer, goes on unweariedly repeating a simple air or theme, now high, now low, in solo, in chorus, ten thousand times reverberated, till it fills earth and heaven with the chant. » (669) De même, la philosophie de Swedenborg prend elle aussi la forme d'images (he saw not abstractly, but in pictures [675]) et de paraboles (When he attempted to announce the law most sanely, he was forced to couch it in parable [675]) qui n'énoncent jamais que la 
même «doctrine » de la mimesis, celle de «l'identité et de l'itération »: «All his figures speak one speech. » (682)

Mais les efforts répétés de Swedenborg pour «proclamer la loi de la manière la plus sensée » sont peut-être moins la marque de son génie et de sa santé mentale - l'indice de la capacité du logos à rendre raison du monde-, que le signe de sa folie mimétique, voire le symptôme de la folie de la mimesis elle-même qui ne se laisse pas encadrer par la logique de l'identité, mais la déborde de tous côtés. Car sous le masque de Mimesis, se cache le dieu Protée que ses métamorphoses rendent insaisissable. Comme le fait ironiquement remarquer Emerson, «The slippery Proteus is not so easily caught. » (676) Moins mimétique que protéiforme, la nature se disperse plutôt qu'elle ne se rassemble sous l'unité d'un concept. Prétendant parler par métaphores, le miméticien ne vise pas autre chose que le sens propre pour ramener la différence à la tautologie de l'identité. Au lieu de laisser filer le sens dans l'écart ouvert par ses tropes poétiques, il cherche à s'approprier la nature pour en faire son double et c'est par ce tour mimétique qu'il entend s'instituer comme sujet afin d'exercer, sur la nature comme sur lui-même, sa souveraineté absolue. Mais pas plus la nature que le sujet ne possèdent de sens propre chez Emerson : «[Nature] is no literalist » (676). Pas de sens littéral, ou propre, mais uniquement des figures qui se substituent les unes aux autres à l'infini ; pas d'original, mais seulement des copies toutes différentes les unes des autres ; pas d'identité, mais toujours déjà de la répétition. Ce qui se découvre ainsi en filigrane, au terme de cette retraversée du platonisme et de ses différents avatars dans Representative Men, c'est que l'arraisonnement de la mimesis et sa subordination à la logique de l'identité ont pour fonction de parer à la menace de la désappropriation, au spectre de la différence et à l'horreur du chaos. De surcroît, cette relecture de l'interprétation platonicienne de la mimesis révèle en creux que l'enjeu de la politique n'est rien d'autre que la question de la souveraineté, du pouvoir et de la domination, et que la cité idéale de Platon a tout d'un État policier ${ }^{5}$. 


\section{La condition mimétique}

Ce n'est toutefois pas pour des motifs politiques, mais bien plutôt esthétiques et littéraires, qu'Emerson se détourne de Swedenborg, car son plus grand crime aura finalement été de ne pas être un poète, mais un «mystique », c'est-à-dire de proposer une théorie des correspondances qui, si elle affirme vouloir rendre raison de la dynamique du monde, se révèle dépourvue de l'énergie vitale qui anime la matière : «Swedenborg's system of the world wants central spontaneity; it [...] lacks power to generate life. » (682) À ce modèle d'une écriture systématique et mortifère, Emerson oppose les Essais de Montaigne où les mots sont «pleins de vaisseaux et de vie » : «Cut these words and they would bleed; they are vascular and alive. »(700) Avec Montaigne, dont le portrait suit immédiatement celui de Swedenborg comme s'il en assurait la relève, c'est la vie qui se présente à même le texte : «Whatever you get here, shall smack of the earth and of real life. » (699) Car tel est bien, selon Emerson, l'enjeu de toute écriture que de sortir de l'imitation mécanique pour accéder à la vie comme telle, n'en déplaise à Platon qui, dans le Phèdre, rapporte l'écriture à une forme figée, une version dégradée de la parole pleine. Là où Platon privilégie la parole sur l'écriture, Emerson reporte la distinction au sein de l'écriture elle-même et sépare le régime mimétique et mensonger du régime poétique et véridique de l'art d'écrire : «This striving after imitative

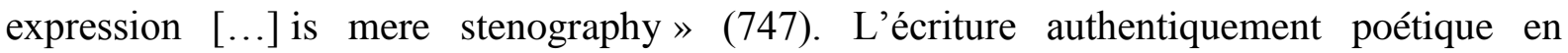
revanche est « une seconde création », et non une création seconde et ancillaire. L'écrivain n'est le «secrétaire » de personne si l'on entend par là un simple copiste ; il a au contraire pour tâche de consigner la vie à l'œuvre : « to report the doings of the miraculous spirit of life that everywhere throbs and works »(746). C'est pourquoi l'écriture doit être elle-même 
génératrice de vie, c'est-à-dire «faire que ce qui est présent soit véritablement présent [...]. L'art n'a pas pour fonction de reproduire le réel ou de produire de l'irréel, il est destiné à attester l'être-présent de ce qui est présent. » (Lacoue-Labarthe, Écrits sur l'art 152) Ou dans les mots d'Emerson : «to supply the axis on which the frame of things turns. » (747) Mais le «propre » du présent, du réel, de la vie, c'est précisément de ne pas se ressembler, d'être toujours « en transition » (682) et de devenir autre. Le «Génie de la vie » répond au nom de «Protée » (the Genius of life, that old cunning Proteus [752]), ce qui revient à dire qu'il n'a pas de nom propre, pas plus qu'il ne possède d'essence propre que l'on pourrait espérer reproduire sur un mode imitatif. Il ne se donne à voir que sous la forme de son retrait, de manière indirecte et oblique : « His very flight is presence in disguise » (752).

À bien des égards, il en va de même de ces représentants dont Representative Men dresse le portrait, ces hommes illustres que le texte offre à l'imitation de tous, mais qui se révèlent, chacun à leur tour, sans identité. Il n’y a en eux rigoureusement rien à imiter, de sorte que la communauté qui se les donne pour référence commune s'édifie au lieu d'une absence. De toutes les figures qui composent le recueil, Shakespeare est peut-être le meilleur exemple, si tant est que le mot convienne encore, d'une telle dépossession antérieure à toute possession de soi. Le chapitre qui lui est consacré multiplie en effet les contradictions et fait vaciller le régime d'oppositions entre l'original et l'imitation, l'authentique et la copie, le propre et l'impropre, la vérité et le mensonge qui ordonnait jusqu'ici la compréhension de la mimesis. Le mouvement tourbillonnant de l'écriture émersonienne vient suggérer que la mimesis est moins seconde que première. Elle s'impose d'avant le sujet et ne relève pas tant de l'imitation d'un original qui n'existe pas que d'un principe d'engendrement infini de figures toutes différentes. L'antériorité de la mimesis dans la constitution de la subjectivité vient alors signifier la configuration originairement plurielle et vicariante de l'identité. 
Certes, cette déconstruction de la mimesis avant l'heure n'apparaît pas d'entrée de jeu, puisque Shakespeare est aussi décrit comme un original indépassable, un créateur à nul autre pareil : «for creation, Shakspeare [sic] is unique. » (722) Son inventivité le distingue à la fois des autres hommes de lettres et du commun des mortels : "Shakspeare is as much out of the category of eminent authors, as he is out of the crowd. » (722) Incomparable, inimitable, c'est un être à part, consacré par la postérité, notamment américaine, qui découvre paradoxalement en lui sa propre image : « he drew the man of England and Europe, the father of the man in America. » (721) Sa puissance d'invention et son génie créateur font de lui le «type » même du poète, c'est-à-dire le modèle en qui tous peuvent se reconnaître et qu'ils doivent aspirer à égaler : «This power of expression [...] makes him the type of the poet » (723). C'est parce qu'il est exceptionnel que le poète est exemplaire ; Shakespeare est représentatif de tous, dans la mesure où il ne ressemble à personne d'autre. C'est en quelque sorte un dieu parmi les hommes auxquels les contemporains d'Emerson vouent une admiration sans bornes, sinon un véritable culte. Mais l'adoration dont il est l'objet interdit de percer son secret et conduit ceux qui s'y adonnent à singer son génie: «Now, literature, philosophy and thought are Shakspearized. His mind is the horizon beyond which, at present, we do not $\operatorname{see}^{6}$. $\gg(718)$ Tragédie de l'imitation où tous sont condamnés à répéter sans les comprendre les paroles du maître anglais.

Mais faire de Shakespeare un obstacle infranchissable contribue simultanément à le revêtir d'un masque impénétrable (Our poet's mask was impenetrable [717-718]) et lui prêter l'épaisseur et l'opacité d'une identité substantielle dont il est en réalité dépourvu. Il n'y a en effet chez lui pas la moindre trace d'ego : « he has no discoverable egotism » $(722)$; «there is no trace of egotism » (724). Le sujet manque à sa place et se constitue sur le fond de ce défaut originaire. Il ne possède aucun caractère propre, ni aucune marque de distinction, rien qui le singularise ou le différencie: «Shakspeare has no peculiarity ${ }^{7}$ » (722). Le Shakespeare 
d'Emerson, comme tout poète, et même tout « sujet », est un « homme sans qualités » dont la grandeur consiste en ceci qu'il n'a rien d'original : «no great men are original » (710); « Great genial power $[\ldots]$ consists in not being original at all » (711).

De cette (in)détermination du sujet découlent deux conséquences quant à la pensée de la mimesis qui s'élabore dans Representative Men. D’une part, le génie apparaît comme un sujet impersonnel et collectif : «It is easy to see that what is best written or done by genius in the world was no man's work, but came by wide social labor » (715). Son œuvre est une entreprise collaborative de tous les instants et s'écrit à plusieurs mains : «Men, nations, poets, artisans, women, all have worked for him » (711). En ce sens, il est un homme de son temps, «a heart in unison with his time and country » (710). Il est même littéralement le produit de son époque, la marionnette de ses contemporains qui lui dictent sa conduite : « he finds himself in the river of the thoughts and events, forced onwards by the ideas and necessities of his contemporaries. » (710) Ses concitoyens parlent à travers lui et sa conscience est un corps conducteur par où passe l'esprit du siècle: «suffering the spirit of the hour to pass unobstructed through the mind» (711). Le poète se fait l'écho de l'intérêt majoritaire, sinon de la volonté générale, et assume son statut d'écrivain public et de ventriloque. Dès le début du chapitre consacré à Platon, Emerson rappelait en effet que chacun est un tissu de citations sans auteur, un palimpseste d'écrits apocryphes : «every man is a quotation from all his ancestors. » (634) Le corps du sujet est un corpus d'œuvres fantômes et l'individu n'accède à lui-même qu'à partir des autres. À l'origine du sujet, il y a toujours déjà citation, répétition, circulation des discours. Ou encore : au commencement, était la mimesis.

D'autre part, et par conséquent, la structure originairement mimétique de la subjectivité autorise toutes les formes de captation d'héritage par un sujet qui, «n'ayant aucune propriété en lui-même [...], est capable de se les approprier toutes.» (LacoueLabarthe, «L'Horreur occidentale » 229) D'où la revendication du plagiat, au motif que le 
« propre » du génie est de savoir s'approprier ses prédécesseurs : «the inventor only knows how to borrow » (634); «the greatest genius is the most indebted man » (710). Le niveau d'endettement est l'indice même de la génialité et à ce jeu, Shakespeare est bien le plus doué de tous, car il est celui qui a contracté le plus grand nombre de dettes à l'égard de ses devanciers : «In point of fact, it appears that Shakespeare did owe debts in all directions, and was able to use whatever he found » (713). S'appuyant sur les travaux d'Edmond Malone, auteur d'une édition critique des œuvres de Shakespeare à la fin du XVIII ${ }^{\mathrm{e}}$ siècle, Emerson met en lumière les emprunts silencieux qui émaillent le corpus shakespearien, tout comme il repère, chez Chaucer, une impressionnante série d'échos venus d'ailleurs, que le poète incorpore et fait discrètement passer pour son œuvre propre. De ce point de vue, la propriété, c'est le vol et le système social qui s'esquisse en pointillés derrière cette compréhension renouvelée de la mimesis est celui de l'appropriation généralisée ou, ce qui revient au même, de la désappropriation généralisée. Personne n'est le propriétaire légitime de quoi que ce soit, et surtout pas de lui-même, et si je peux à ce titre prétendre m'attribuer les propriétés qui me manquent, je risque également à tout instant d'être dépossédé de tout. Poussée jusqu'à son terme, la logique de cette mimesis productive, dont le sujet n'est finalement que l'effet, aurait ainsi pour conséquence une désorganisation sans précédent de l'espace social et politique.

\section{Le partage des incommensurables}

Il s'agirait donc de contrôler, sinon de résorber, la différence originaire à partir de laquelle se (dé)constitue le sujet en tant que sujet mimétique et qui se tient au principe du bouleversement de l'ordre politique qui menace. Aussi n'est-il pas indifférent que le portrait de Napoléon suive immédiatement celui de Shakespeare - la correspondance nous apprend 
même que c'est en rédigeant ce texte qu'Emerson commence à préparer le cycle de conférences qui donnera Representative Men, auquel il devait initialement servir de conclusion $^{8}$. Certains passages du recueil assimilent en effet par un coup de force la différence du représentant à une marque de distinction qui serait de nature à légitimer son pouvoir souverain. Ce dernier prend alors les traits d'une figure divine chargée de transfigurer le chaos du monde en un ordre apaisé : «a god leading things from disorder into order » (659). «It is for man to tame the chaos » (632), affirme Emerson en conclusion du premier chapitre, suggérant que c'est là l'usage principal du grand homme, sa fonction première au sein de la communauté. Cette perspective ne manque jamais de séduire, au moins pour un temps, le patricien de Nouvelle-Angleterre, soupçonneux de la démocratie populaire, qui sommeille en Emerson. Dès la première page du texte, il évoque ainsi, avec un dégoût quasi malthusien, le spectre d'une populace parasite, trop contente de profiter de la charité commune: «But enormous populations, if they be beggars, are disgusting, like moving cheese, like hills of ants, or of fleas - the more, the worse. » (615) La tentation de l'autoritarisme est le contrecoup de cette aversion pour le peuple, mais elle conduit à une distorsion, et même une confiscation, de la représentation par un représentant qui, s'arrogeant le droit de parler au nom des autres, les réduit au silence et ne représente finalement que luimême, dans sa différence d'avec une communauté qu'il soumet à son pouvoir absolu. Retour insidieux de l'idéal platonicien du philosophe-roi dictant sa loi à la multitude qui vient s'incarner dans l'image de Napoléon façonnant ses généraux dans la boue du champ de bataille (738).

Toutefois, très tôt dans le chapitre liminaire, cette conception aristocratique de la représentation était rejetée pour n'être que la rançon d'une faiblesse de la volonté : «The imbecility of men is always inviting the impudence of power. [...] But the true genius seeks to defend us from itself. » (623) Chez les faibles d'esprit, coupables de se laisser obnubiler 
par l'éclat trompeur du représentant, le respect de l'autorité se dégrade en fétichisme idolâtre de la personne qui en est le dépositaire (idolatry of the herald [623]). Mais le génie est à luimême son propre antidote et invite à se protéger du risque qu'il porte en lui, car il enjoint de se détourner rapidement de lui pour le remplacer par un autre appelé à subir le même sort. Ou du moins est-ce ainsi qu'Emerson naturalise le système l'égale rotation des représentants théorisés par James Harrington dans The Commonwealth of Oceana en 1656, qui assure à chacun le droit d'accéder à la représentation et donc de prendre part au gouvernement : «Rotation is [nature's] remedy. The soul is impatient of masters and eager for change. [...] Rotation is the law of nature. » (623) Cette provision semble aussi éminemment américaine, puisque c'est à cet endroit que sont convoqués Franklin, Jefferson et Jackson, qui aura troqué son uniforme de général pour le costume de Président, soit trois des rares exemples autochtones que donne le texte :

When nature removes a great man, people explore the horizon for a successor; but none comes, and none will. His class is extinguished with him. In some other and quite different field the next man will appear; not Jefferson, not Franklin, but now a great salesman, then a road-contractor, then a student of fishes, then a buffalo-hunting explorer, or a semi-savage Western general. (623)

La structure du recueil lui-même vient mimer le mouvement qu'il décrit, dans la mesure où chacun des chapitres fait l'éloge du héros qui lui donne son titre avant de procéder à sa critique et de lui substituer le suivant. Contre-pouvoir à «l'excès d'influence » du grand homme, l'alternance perpétuelle des représentants assurerait, par une juste répartition des places au sein du gouvernement, l'équilibre des forces dans la communauté : «We balance one man with his opposite, and the health of the state depends on the see-saw. » (628) 
Mais dans cette perspective, l'espace démocratique deviendrait inévitablement le lieu d'une mimesis sans fin, d'une sempiternelle substitution de figures représentatives que plus rien ne permet de distinguer. Comme Tocqueville, pour qui la démocratie américaine conduit au conformisme et à l'indistinction, Emerson laisse entendre qu'une démocratie qui se pense et se forme sur le modèle de la «rotation » conduit à l'arasement des différences, car elle repose sur le principe de l'équivalence générale. Ainsi entendue, la démocratie correspond littéralement au règne de la médiocrité et porte en elle l'avènement de ce que le statisticien belge Adolphe Quételet appelle en 1835, dans son essai Sur l'homme, «l'homme moyen ». Or Emerson a connaissance des travaux de Quételet, qu'il croit être français (Packer), et son intérêt pour un héros «moyen » transparaît à plusieurs reprises dans Representative Men. De Platon, il écrit notamment, dans une formule paradoxale, « he is a great average man » (644), comme si sa grandeur se mesurait à sa capacité à être représentatif de la moyenne. D’où aussi la fascination pour le scepticisme, cette philosophie qui consiste à occuper ce que Montaigne appelle « la moyenne région », «the middle ground» dans la traduction d'Emerson (693). Le privilège de la moyenne se reflète même dans l'architecture du recueil, puisque le chapitre sur Montaigne y occupe la position médiane. De ce point de vue, on peut dire de la démocratie mimétique et « rotative » d'Emerson qu'elle est une démocratie de la statistique, ou plutôt de la «Staatistik », du nom que donne à cette discipline l'économiste et jurisconsulte allemand Gottfried Achenwall et qui, étymologiquement, assigne pour horizon à la conduite de l'État l'avènement de la moyenne arithmétique.

Comme le rappelle cependant Jean-Luc Nancy, si «le monde démocratique s'est développé dans le contexte - auquel il est lié d'origine - de l'équivalence générale », cette expression désigne aussi bien « la réduction des excellences dans la médiocrisation » que «la monnaie et la forme marchande, c'est-à-dire le cour du capitalisme » (Nancy, 44). Or le portrait de Napoléon explicite ce lien qui unit la démocratie au capitalisme (Niemeyer). 
Napoléon est en effet présenté à la fois comme l'incarnation du principe démocratique («the incarnate Democrat » [727], «this pattern democrat » [730]) et le défenseur de la loi du marché, le représentant des forces de l'économie : «Paris and London and New York, the spirit of commerce, or money and material power, were also to have their prophet; and Bonaparte was qualified and sent. » (728) Pour ce général d'armée et capitaine d'industrie en puissance, la concurrence est la seule vertu possible. En ce sens, la démocratie de l'équivalence générale ne diffère pas du règne de la rivalité mimétique où chacun copie son voisin pour mieux s'enrichir à ses dépens, et c'est bien un tel système que décrit non sans regret Emerson au début du recueil :

Here is great competition of rich and poor. We live in a market, where is only so much wheat, or wool, or land; and if I have so much more, every other must have so much less. I seem to have no good without breach of good manners. Nobody is glad in the gladness of another, and our system is one of war, of an injurious superiority. Every child of the Saxon race is educated to wish to be first. It is our system; and a man comes to measure his greatness by the regrets, envies and hatreds of his competitors.

Dans cette guerre de chacun contre tous, seul prime l'intérêt particulier, seule compte la loi du plus fort. L'espace public se réduit à la sphère économique et ressemble à un gigantesque champ de bataille jonché de cadavres, tandis que l'activité politique se résume à l'accumulation de richesses matérielles qui deviennent les insignes du pouvoir.

$\grave{A}$ cet endroit du texte, Emerson envisage toutefois une autre forme de concurrence qui ne serait pas indexée sur l'appât du gain et la recherche du pouvoir, mais où chacun se donnerait pour fin l'excellence désintéressée, c'est-à-dire la vertu (arêtè) : 
I find him greater when he can abolish himself and all heroes, by letting in this element of reason, irrespective of persons, this subtilizer and irresistible upward force, into our thought, destroying individualism, the power so great that the potentate is nothing. Then he is a monarch who gives a constitution to his people; a pontiff who preaches the equality of souls and releases his servants from their barbarous homages; an emperor who can spare his empire. (625-626)

Le modèle de citoyenneté auquel aspire Emerson est celui d'un empereur sans empire, qui ne vise ni son profit ni le pouvoir. La sphère publique n'est plus la scène d'affrontements mimétiques, mais le théâtre d'une «émulation » vertueuse entre tous les citoyens, l'effort de chacun pour exceller devant ses pairs invitant les autres à faire de même : «great men exist that there may be greater men » (632); «We are emulous of all that man can do » (620). L'enjeu de la politique revient alors à se distinguer des autres sans établir de hiérarchie, et permettre à tous d'en faire autant.

Ce que suggère là Emerson n'est pas sans affinités avec la manière dont Hannah Arendt détermine l'espace public comme le lieu de l'excellence commune, le théâtre d'un agon où les citoyens entreprennent librement de se distinguer les uns par rapport aux autres et, par leur action, engagent la communauté ${ }^{9}$. Mais on peut également le rapprocher du « défi » que Jean-Luc Nancy assigne à la démocratie et qui consiste à produire l'égalité, c'est-à-dire paradoxalement à ouvrir à la possibilité de l'«inéquivalence », et ainsi permettre l'«affirmation de la valeur incommensurable » de chacun, car « la stricte égalité est le régime où se partagent ces incommensurables » (Nancy 47). Chez Emerson, comme chez Arendt et Nancy, «la démocratie est aristocratie égalitaire » (Nancy 61). Tel est peut-être, en dernière analyse, le sens de la mimesis qui s'opère dans Representative Men, où la succession des 
hommes représentatifs offre à chacun une image de la différence à laquelle l'idéal démocratique l'autorise à prétendre. De «Plato; or, the Philosopher » à «Goethe; or, the Writer », les titres des chapitres qui composent le recueil viennent mettre en évidence, dans l'écart entre les deux termes de la copule, cette politique de l'inéquivalence. Au bout du compte, la représentation de la différence, la représentation comme différence, permet de fonder la communauté, non pas sur le pouvoir autoritaire ou l'équivalence des ressemblances, mais bien sur une égalité d'accès à la distinction, dans l'imitation de ce qui reste inimitable.

\section{Ouvrages cités}

Arendt, Hannah. La Condition de l'homme moderne. Paris : Presses Pocket, 1988.

Constantinesco, Thomas. Ralph Waldo Emerson. L'Amérique à l'essai. Paris : Éditions Rue d'Ulm, 2012.

DERRIDA, Jacques. « Envoi » (1980). Psyché. Inventions de l'autre. Paris : Galilée, 1998.

EMERSON, Ralph Waldo. Representative Men. Emerson: Essays and Lectures. Ed. Joel Porte. New York: The Library of America, 1983. 612-761; The Letters of Ralph Waldo Emerson. Ed. Ralph L. Rusk et Eleanor M. Tilton. New York : Columbia UP, 1939-1995, 10 vol.

Lacoue-Labarthe, Philippe. «Typographie». Mimesis des Articulations. Paris : AubierFlammarion, 1975. 165-270 ; «L'Horreur occidentale» (1996). Lignes 22 (mai 2007) : 224234 ; «Sur le "Théâtre des réalités" ». Écrits sur l'art. Genève : Les Presses du réel, 2009. $147-157$.

NANCY, Jean-Luc. Vérité de la démocratie. Paris : Galilée, 2008.

NIEMEYER, Mark. «Emerson's Napoleon; or, the French Emperor as (American) Democrat and Businessman ». Sources 18 (Spring 2005) : 29-41. 
PACKER, Barbara. «Emerson and the Terrible Tabulations of the French ». Transient and Permanent: The Transcendentalist Movement and Its Contexts. Ed. Charles Capper and Conrad Edick Wright. Boston : Historical Society and Northeastern UP, 1999. 148-167.

Platon. République. Paris : Garnier-Flammarion, 1991 ; Le Banquet, Phèdre. Paris : GarnierFlammarion, 1992 [1964].

QUETELET, Adolphe. Sur l'homme. Paris : Fayard, 1991 [1835].

RANCIERE, Jacques. Aisthesis. Scènes du régime esthétique de l'art. Paris : Galilée, 2011.

SCHULZ, Dieter. «Ralph Waldo Emerson : From viri illustres to Representative Men ». Ed.

Dorothea Walz. Scripturus Vitam: Lateinische Biographie von der Antike bis in die Gegenwart. Heidelberg : Mattes Verlag, 2002. 1185-1195.

${ }^{1}$ Cette étude poursuit la réflexion que j'ai entamée dans L'Amérique à l'essai sur la représentation littéraire et politique chez Emerson (222-241) ; je me permets d'y renvoyer pour une mise en contexte de la problématique et un état de la critique consacrée à Representative Men. Les références au texte d'Emerson sont tirées de l’édition « Library of America ».

2 On reconnaîtra ici ce que cette étude doit à l'entreprise de déconstruction de la mimesis telle que l'ont proposée Philippe Lacoue-Labarthe et Jacques Derrida qui chacun mettent en lumière la double valence du mot « représentation », lequel signifie à la fois reproduire et rendre présent. Je suis également redevable à Bernard Baas pour sa lecture de la tradition philosophique, de Platon à Hannah Arendt et au-delà.

${ }^{3}$ Dans Aisthesis, Rancière entreprend de lire « The Poet » comme une telle opération de partage où « la tâche du poète peut s'identifier avec la construction d'une communauté » (89). 
${ }^{4}$ James John Garth Wilkinson (1812-1899) est un médecin et homéopathe anglais, grand ami de Henry James Sr. Il assure la première édition populaire des Songs of Innocence and Experience de Blake avant de s'atteler à la traduction des œuvres de Swedenborg.

${ }^{5}$ Telle était déjà la critique d'Aristote qui récusait l'idée que la politique serait affaire de pouvoir et pourrait être prédiquée sur un savoir détenu par le philosophe-gouvernant. Ce qui revient à dire que la lecture émersonienne de la mimesis est elle-même prise dans le circuit de la répétition.

${ }^{6}$ L'influence excessive de Platon et de Swedenborg est dénoncée au moyen de néologismes similaires : Platonise (659), Swedenborgise (682).

${ }^{7} \mathrm{Si}$ cette formule signifie d'abord que Shakespeare n'est pas difficile, que tout lui convient, impossible de ne pas entendre l'aveu de désappropriation de soi qui vient s'y dire par la bande.

${ }^{8}$ Dans une lettre du 29 septembre 1845, Emerson affirme : « it was the first written, though it should be last in the course » $(L, 3: 305)$.

${ }^{9}$ Faute de place, nous ne pouvons ici que renvoyer aux analyses d'Arendt, notamment dans La Condition de l'homme moderne. Une phrase d'Emerson prend ainsi une résonance singulière : « No man, in all the procession of famous men, is reason or illumination of that essence we were looking for; but is an exhibition, in some quarter, of new possibilities. » (630) Chez l'un comme chez l'autre, la politique ne consiste plus à accomplir une essence, car d'essence l'homme n'a point, mais à faire usage de la liberté que lui confère ce manque ontologique pour se porter au-delà de lui-même dans l'action, s'ouvrir à de nouvelles possibilités et, ce faisant, laisser une marque dans la mémoire de la communauté. Ce serait aussi une autre manière de comprendre l'idée d'un " grand homme moyen », sorte de héros ordinaire, et de rejoindre Stanley Cavell qui envisage le transcendantalisme émersonien comme une philosophie et une politique de l'ordinaire 